\title{
Editorial: Annals of Mathematics and Artificial Intelligence special issue on multi-robot coverage, search, and exploration
}

\author{
Gal A. Kaminka - Amir Shapiro
}

Published online: 4 March 2009

(C) Springer Science + Business Media B.V. 2009

Research in multi robot area coverage, search, and exploration has been receiving consistent attention in recent years, due to the increasing number of real-world applications, such as vacuuming, lawn mowing, demining, surveillance, search and rescue operations, mapping, planetary exploration, etc. All of these applications require that the area of interest be covered by the robots sensors or end-effectors for various purposes. The use of multiple robots potentially provides redundancy and offers opportunities for increasing efficiency. The problem of multi robot area coverage imposes great challenges to researchers in robotics and AI area. This special issue explores the new research frontiers that emerge as new applications are identified and new technologies in robots are introduced. This special issue follows in the footsteps of the highly successful 2001 special issue on coverage. However, the objective of this issue has been broaden to include the adjacent and related fields of multi-robot search and multi-robot exploration.

This special issue contains nine research papers that were carefully selected to be both of high quality and to deal with a wide verity of problems related to multi-robot coverage, search, and exploration. The first paper by Rekleitis et al. concerns with the multi-robot coverage problem. The paper presents an algorithm that solves the problem online by sending scout robots to explore the area. The second paper by Agmon, Hazon, and Kaminka deals with the same problem, but here the authors took a different approach by circumnavigating a spanning tree. The third paper by Sarid and Shapiro analyze the competitive complexity of a multi-robot search

G. A. Kaminka (凶)

Computer Science Department, Bar Ilan University, Ramat Gan 52900, Israel e-mail: galk@cs.biu.ac.il

\footnotetext{
A. Shapiro Mechanical Engineering Department, Ben Gurion University of the Negev, P.O. Box 653, Beer Sheva 84105, Israel e-mail: ashapiro@bgu.ac.il
} 
problem. The fourth paper by Stachniss, Mozos, and Burgard uses place-type labels to explore an office like environment by group of robots. The fifth paper by Vincent et al. describes an integrated experimental setup of hundred robots for exploration and mapping. The sixth paper by Lee and Chong describes a method to deploy a robot swarm to construct sensor network with uniform spatial density. The seventh paper by Gasparri, Krishnamachari, and Sukhatme discusses coverage using sensor network. The eighth paper by Amstutz, Correll, and Martinoli describes an algorithm for boundary coverage by group of robots. Finally the last paper by Sujit and Beard describes exploration by group of UAVs.

Together, these nine articles present a good cross-section of the field and show various aspects of the multi-robot coverage, search, and exploration problem. All the papers presents unique innovative multi-robot cooperation algorithms, and most of the papers also demonstrate their results in a fully functional real-world robotic experimental setup. We hope that you find these articles interesting, inspiring and motivating. Finally, we would like to thank the editor-in-chief Prof. Martin C. Golumbic for giving us the opportunity to put together this special issue. We thank all the authors, and the reviewers for their fine efforts and contributions. 